\title{
Extraoral Facial Pain Caused by an Intraoral Stimulus: A Case Report
}

ISSN: 2637-7764

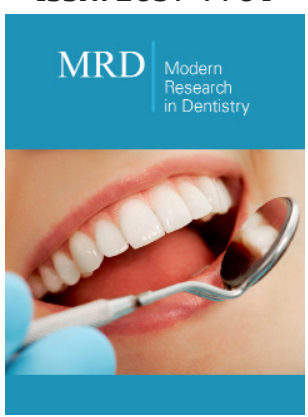

*Corresponding author: Talal H Salame, Department of Prosthodontics, Faculty of Dentistry, Hadath Campus, The Lebanese University, Beirut, Lebanon

\section{Submission: 眥 August 26, 2021}

Published: 皆 September 14, 2021

Volume 6 - Issue 5

How to cite this article: Talal $\mathrm{H}$ Salame. Extraoral Facial Pain Caused by an Intraoral Stimulus: A Case Report. Mod Res Dent. 6(5). MRD. 000647. 2021. DOI: 10.31031/MRD.2021.06.000647

Copyright@ Talal H Salame, This article is distributed under the terms of the Creative Commons Attribution 4.0 International License, which permits unrestricted use and redistribution provided that the original author and source are credited.
Talal H Salame*

Department of Prosthodontics, Faculty of Dentistry, Hadath Campus, The Lebanese University, Beirut, Lebanon

\begin{abstract}
Dentists are faced daily by many pain conditions. Some are common and easy to deal with such as dental pain and others are more complicated. The pain could originate intraorally and/or extraorally. While diagnosing and treating intraoral pain seems to be an easy task for an experienced dental practitioner, the diagnosis and management of facial pain can be very difficult as presentation of many orofacial pain conditions could overlap and the diagnosis could be complicated by more than one obstacle. An adequate diagnosis could be achieved by a careful history taking and thorough clinical examination. If the origin of pain is clear, the task for the clinician could be achieved easily. However, in the absence of a clear cause or origin of the pain, diagnosis becomes diffuse and extra care should be taken and thinking must overpass the borders of the traditional square. This case offers a brief discussion of an extraoral pain condition that is exacerbated by an intraoral lesion.
\end{abstract}

Keywords: Orofacial pain; Intraoral pain; Extraoral pain; Aphtous ulcers; Herpes; Varicella zoster; Autoimmune condition

\section{Introduction}

Pain is the primary cause for patients to seek medical advice. It is defined as "an unpleasant sensory or emotional experience associated with actual or potential tissue damage or described in terms of such damage" [1]. The types of the pain could change according to different factors including pathophysiology such as inflammatory or traumatic, location such as visceral or neurological or muscular, and the trigger such as functional or spaontaneous [2], amongst others.

Unlike temperature, blood pressure, and other vital information, there is no evidence based, well standardised tools that help in diagnosing pain accurately. By far, the only useful tool used broadly by practitioners is verbal description. But due to its affective component and subjective nature [3], pain language becomes diffuse and distinction between different qualities of pain turns out to be difficult for the patient to express and for the practitioner to understand and thus diagnosis becomes a dilemma [4,5].

Pain occurs whenever a thermal, chemical and/or mechanical, tissue-damaging, stimulus takes place. Specialized central neural pathways carry information about the location, the intensity and the quality of the stimulus from different peripheral nociceptors to the central nervous system where the information are analysed [6]. However, the site of pain does not always coincide with the site of pathophysiology [7], and pain could manifest in an area that is not related to the area of the original stimulus and this is termed "referred pain". In this instance, the Pain could occur in either area or in both areas at the same time [8].

The term orofacial pain includes a wide range of muscular, vascular, neurological and/ or psychological conditions. Studies show that the mouth and the face are some of the most common areas that patients report pain $[9,10]$, with $81.4 \%$ of myofascial pain sufferers reporting pain in the head and face. It could be associated with intraoral anatomical structures, such as the teeth, the palate, the tongue or any other intraoral structure or 
extraoral components such as the face, the head, the neck, the temporomandibular joints and others. It could comprise common headaches, serious neoplastic conditions, advanced autoimmune disorders and many other conditions [11].

This paper discusses an acute clinical presentation of an extraoral facial pain exacerbated by an intraoral condition.

\section{Clinical presentation}

\section{Chief complaint and history}

A 49-year-old male presented to the surgery with complaint of persistent pain in the left side of the face towards the zygomatic process. The pain is continuous and started around 2 weeks ago as a burning sharp sensation. It has increased gradually and could be exacerbated by simple touch.

\section{Clinical examination}

Extraoral examination revealed no abnormalities. The patient evaluated the pain as 5 out of ten on the Visual Analogue Scale
(VAS). The pain increased on manual palpation of the painful area, that stretches from the right side of the nose all the way under the left eye on the zygomatic arch and up to the distal border of the left eye on the frontal process of the zygomatic bone. The patient did not have similar pain previously and there was no clear cause in his history that could explain such pain. However, he declared that a painful lesion has appeared in his mouth days earlier. As so, the clinician proceeded to the intraoral examination.

\section{Intraoral examination}

Cluster of punctate red lesions were found on the left half of the patient's hard palate (Figure1). Further questioning revealed that the patient used to have many lesions in his oral cavity at different occasions and that this has been going for a long period of time. Other lesions were occurring in different areas of his mouth with differences in shapes and sizes. Some of them presented with large white ulcerative areas of few millimetres accompanied with severe pain.

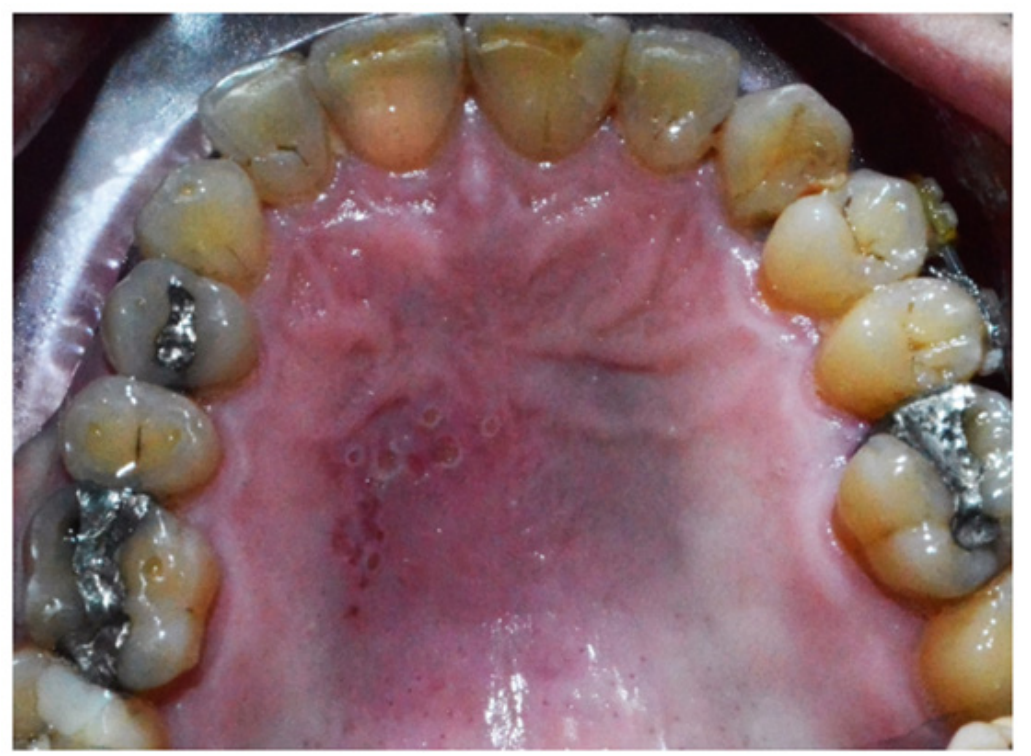

Figure 1: Cluster of punctate red lesions on the left half of the hard palate (picture mirrored during photography).

\section{Discussion}

Many conditions could cause punctate lesions in the oral cavity. This includes drug reactions, contact, allergy, trauma, fungal and viral infections, autoimmune conditions and immunodeficiency [12]. There was no history of trauma, allergy, drug intake or immunodeficiency for this patient. The presence of cluster ulcers on the hard palate (fixed mucosa) suggested a herpetic lesion. The recurrence of the presentation is consistent with the common inflammatory disease recurrent herpetic stomatitis. But previous presentations with large, very painful lesions could suggest aphtous ulcers. The lack or the presence of a preliminary vesicular phase could help in distinguishing between the two lesions [12].

The distribution of the vesicles along the posterior dental branch of the maxillary nerve could point towards the human herpes virus 3 infection or herpes zoster. This could occur after a primary infection by chickenpox where the varicella-zoster virus attains the gasserian ganglion and remains latent. When a secondary activation occurs later in life, a cluster of unilateral painful vesicular lesions appear along one of the distributions of the maxillary or the mandibular nerves (herpes zoster, or shingles), especially on the palate, accompanied with fever and lymphadenopathy [12] which were not present in this patient.

The presence of other lesions in different parts of the body could suggest more sinister condition. Many autoimmune diseases could present with oral manifestations such as rheumatoid arthritis, inflammatory myopathies, systemic lupus erythematous, systemic sclerosis, Sjögren's syndrome and Behcet syndrome [13,14]. Behcet syndrome in particular presents with painful mucosal and/ 
or cutaneous lesions that could occur in the oral cavity, the skin, the eye and the genital area [15]. Oral lesions usually occurs before the systemic presentation that could include joints pain and the involvement of the gastrointestinal system, the cardiovascular system and the nervous system [16]. A close relative of the patient was diagnosed with this condition few years earlier.

Pain is signalled by specialized central neural pathways carrying information about stimulus quality, intensity and location from different peripheral nociceptors [6]. Nociceptors are mostly free nerve endings and respond usually to painful stimuli. Under abnormal conditions such as trauma or inflammation, the release of algesic substances like bradykinin, prostaglandin and serotonin from the terminals of thin afferent fibres cause sensitisation of adjacent nociceptors [17]. "Referred pain" occurs when the site of the pain does not coincide with the site of pathophysiology [7]. Afferent from different parts of the body could converge into the same nervous pathway or the same area in the central nervous system [18]. This convergence is considered to be an integral mechanism underlying hyperalgesia, poor localisation, and referred pain that are all common in the orofacial area [18].

The extraoral facial pain of the patient was referred from the intraoral lesions. Through its three branches, the zygomatic, the pterygopalatine (or sphenopalatine), and the posterior superior alveolar nerves, the maxillary nerve carries sensory information from the lower eyelid, the lateral wall of the orbit, the zygoma, the cheek, the nares, the upper lip, the upper teeth and gums, the nasal mucosa, the palate and roof of the pharynx, the maxillary, ethmoid and sphenoid sinuses, and parts of the meninges [19]. Any area of these could be affected by pain that could originate from any other area that belongs to the same nerve.

\section{Conclusion}

Extraoral pain could be exacerbated by intraoral causes which could complicate the diagnosis of orofacial pain. The need for a complete and careful history and the use of thorough examination procedures is essential in order to uncover the intraoral causes. Dentists are supposed to demonstrate the ability to act in the early diagnosis and management of these disorders to fulfil the patients' urgent medical needs.

\section{References}

1. (2011) IASP (International Association for the Study of Pain). Pain terms.

2. Woolf CJ (2004) Pain: moving from symptom control toward mechanismspecific pharmacologic management. Ann Intern Med 140(6): 441-451.
3. Rainville P, Bushnell MC, Duncan GH (2001) Representation of acute and persistent pain in the human CNS: potential implications for chemical intolerance. Ann NY Acad Sci 933(1): 130-141.

4. Hawthorn J, Redmond K (1998) Pain: causes and management. Blackwell Science Ltd, Oxford, England.

5. Mauro G, Tagliaferro G, Montini M, Zanolla L (2001) Diffusion model of pain language and quality of life in orofacial pain patients. J orofac pain 15(1): 36-46.

6. Dubner R (1985) Recent advances in our understanding of pain. In: Klineberg I, Sessle BJ (Eds.), Orofacial pain and neuromuscular dysfunction: mechanisms and clinical correlates. Pergamon Press, Oxford, England.

7. Melzack R, Wall PD (1999) Textbook of pain. Churchill Livingstone, Edinburgh, London, UK.

8. Galea MP (2002) Neuroanatomy of the nociceptive system. In: Strong J (Ed.), Pain: a textbook for therapist. Churchil Levingstone, London, UK.

9. Sessle BJ (2008) Orofacial pain: from basic science to clinical management: the transfer of knowledge in pain research to education, Quintessence Pub, Chicago, Illinois, USA.

10. Carlsson GE, LeResche L (1995) Epidemiology of temporomandibular disorders. In: Sessle BI, Bryant PS, Dionne RA (Eds.), Temporomandibular disorders and related pain conditions. IASP Press, Seattle, USA.

11. McGregor N, Butt H, Zerbes M, Klineberg I, Dunstan R, et al. (1996) Assessment of pain (distribution and onset), symptoms, scl-90 inventory responses, and the association with infectious events in patients with chronic orofacial pain. J Orofacl Pain 10(4): 339-350.

12. Lawson W (2012) Punctate oral erosions: Self-limited "sore" - Or something more serious? Consultant 52(10): 669-678.

13. Klasser GD, Balasubramaniam R, Epstein J (2007) Topical reviewconnective tissue diseases: orofacial manifestations including pain. J Orofac Pain 21(3): 171-184.

14. Abrão AL, Santana CM, Bezerra AC, Amorim RF, Silva MB, et al. (2016) What rheumatologists should know about orofacial manifestations of autoimmune rheumatic diseases. Rev Bras Reumatol S0482-5004(16): 00021-00028.

15. Schreiner DT, Jorizzo JL (1987) Behcet's disease and complex apthosis. Dermatol Clin 5(4): 769-778.

16. Akdeniz N, Elmas ÖF, Karadağ AS (2019) Behçet syndrome: A great imitator. Clin Dermatol 37(3): 227-239.

17. Mense S, Simons DG (2001) Muscle pain: Understanding its nature, diagnosis, and treatment. Lippincott Williams and Wilkins, Ottawa, Canada.

18. Sessle BJ (2000) Acute and chronic craniofacial pain: brainstem mechanisms of nociceptive transmission and neuroplasticity, and their clinical correlates. Crit Rev Oral Biol Med 11(1): 57-91.

19. Boileau JC (1962) Atlas of anatomy. Baltimore, Williams \& Wilkins, USA. 